\title{
FLEXIBLE NILSTABLE ALGEBRAS
}

\section{LOUIS A. KOKORIS}

1. Introduction. In the study of finite dimensional simple flexible power-associative algebras, one of the residual classes of algebras is the class of degree two. These are simple power-associative algebras satisfying the flexible identity $(x y) x=x(y x)$ and with a unity element $1=u+v$ which is the sum of two orthogonal absolutely primitive idempotents $u$ and $v$.

It is known (see $[1 ; 3]$ ) that such an algebra $A$ over a field $F$ whose characteristic is not 2 can be decomposed relative to $u$ and written $A=A_{1}+A_{12}+A_{2}$ where $A_{1}=A_{u}(1)=A_{v}(0), \quad A_{12}=A_{u}(1 / 2)$ $=A_{v}(1 / 2), A_{2}=A_{u}(0)=A_{v}(1)$ and $x$ is in $A_{u}(\lambda)$ if and only if $x u+u x$ $=2 \lambda x$. It is also known [1] that $x u=u x=\lambda x$ if $\lambda=0,1$, that $A_{1}$ and $A_{0}$ are orthogonal subalgebras, that $x u$ and $u x$ are in $A_{12}$ if $x$ is in $A_{12}$, and we have the multiplicative properties

$$
\begin{array}{ll}
A_{12} A_{1} \subseteq A_{12}+A_{2}, & A_{1} A_{12} \subseteq A_{12}+A_{2}, \\
A_{12} A_{2} \subseteq A_{12}+A_{1}, & A_{2} A_{12} \subseteq A_{12}+A_{1}
\end{array}
$$

Furthermore [2;6], $A_{1}=u F+N_{1}, A_{2}=v F+N_{2}$, where $N_{1}$ and $N_{2}$ are nil subalgebras of $A_{1}$ and $A_{2}$, respectively. The algebra $A$ is called $u$-nilstable (or nilstable with respect to $u$ ) if $A_{12} A_{i} \subseteq A_{12}+N_{3-i}$ and $A_{i} A_{12} \subseteq A_{12}+N_{3-i}$ for $i=1,2$, and $A$ is called nilstable if it is $u$ nilstable for every idempotent $u$. Also $A$ is said to be $u$-stable if $A_{12} A_{i} \subseteq A_{12}$ and $A_{i} A_{12} \subseteq A_{12}$ for $i=1,2$ and stable if it is $u$-stable for every idempotent $u$.

Oehmke has shown [6] that if $A$ is stable over an algebraically closed field $F$, it has the property that the associated algebra $A^{+}$ (which is the same vector space as $A$ but has product $x \cdot y$ $=(1 / 2)(x y+y x)$ where $x y, y x$ are products in $A)$ is the simple Jordan algebra of degree 2 . We shall obtain this result for nilstable algebras and then we shall completely determine all flexible algebras of degree 2 over an algebraically closed field which have the property that $A^{+}$ is the simple Jordan algebra of degree 2. It seems desirable to study the nilstable algebras since it is easy to show that an algebra of characteristic zero is nilstable.

The proof will depend on [4] where it was proved that over an algebraically closed field of characteristic $\neq 2,3$, a nilstable simple commutative power-associative algebra is Jordan. This result is not 1961.

Presented to the Society, December 12, 1960; received by the editors March 27, 
stated in reference [4] but is implied by the proof. The author is confident that the results of this paper could with some additional effort be proved for characteristic 3 , but this effort does not seem worthwhile.

2. Proof that $A^{+}$is Jordan. It is assumed throughout this paper that $A$ is a nilstable simple flexible power-associative algebra of degree 2 over an algebraically closed field of characteristic $\neq 2,3$. This implies that $A^{+}$is a nilstable commutative power-associative algebra of degree 2 . Since $A$ is flexible it satisfies

$$
(x y) z+(z y) x=x(y z)+z(y x),
$$

which is the linearized form of the flexible law.

Theorem 1. A flexible power-associative algebra $A$ is $u$-nilstable if and only if $A^{+}$is u-nilstable.

It is clear that $A^{+}$is $u$-nilstable if $A$ is $u$-nilstable. Conversely, let $A^{+}$be $u$-nilstable and let $y=u$ in (1) to obtain $(x u) z+(z u) x=x(u z)$ $+z(u x)$. If $x$ is in $A_{1}$ and $z$ in $A_{12}$, we know that $x u=u x=x, u z=z-z u$, and $z u, u z$ are in $A_{12}$. It follows that $x z+(z u) x=x z-x(z u)+z x$ or $z x=(z u) x+x(z u)$. Since $(z u) x+x(z u)=2(z u) \cdot x$ and since $A^{+}$is $u$ nilstable, $z x$ is in $A_{12}+N_{2}$. The product $x z=2 x \cdot z-z x$ is also in $A_{12}$ $+N_{2}$. The proof is similar if $x$ is in $A_{2}$.

CoRollary. A flexible power-associative algebra $A$ is nilstable if and only if $A^{+}$is nilstable.

Theorem 2. A flexible power-associative algebra of degree 2 and characteristic zero is nilstable.

This result follows from the corollary to Theorem 1 and from Theorem 6 of [3] which states that a commutative power-associative algebra of characteristic zero is nilstable.

ThEOREM 3. Let $A$ be a nilstable simple flexible power-associative algebra of degree 2 over an algebraically closed field of characteristic $\neq 2,3$. Then $A^{+}$is a Jordan algebra. ${ }^{1}$

The proof is made by leaning heavily on [4] where it is shown that $A^{+}$is Jordan if it is also simple. In that reference simplicity is used in two places. First in the proof of Lemma 7 when the ideal $A_{12}+N_{1}+N_{2}$ is constructed and, second, at the end when the ideal $A_{12}\left(N_{1}+N_{2}\right)$

\footnotetext{
${ }^{1}$ By using a generalization of [4] it is possible to state Theorem 3 in a more general form. See the author's On nilstable algebras, Proc. Amer. Math. Soc. 9 (1958), 697701.
} 
$+N_{1}+N_{2}$ is constructed. Both times simplicity is used to set the ideal equal to zero. We cannot do that here but we do use the information concerning the ideals of $\mathrm{A}^{+}$.

Lemma 1. If $x y+y x$ and $x(y u)+(y u) x$ are in $N=N_{1}+N_{2}$ for $x, y$ in $A_{12}$, then $x y$ and $y x$ are in $N+A_{12}$.

Write $x y=\alpha u+\beta v+n_{1}+n_{2}+a_{12}$ where $\alpha, \beta$ are in $F, n_{1}$ in $N_{1}, n_{2}$ in $N_{2}, a_{12}$ in $A_{12}$. Then $y x=-\alpha u-\beta v+q_{1}+q_{2}-a_{12}$ with $q_{1}$ in $N_{1}, q_{2}$ in $N_{2}$. By (1), $(x y) u+(u y) x=x(y u)+u(y x)$. Add $(y x) u$ to both sides of this equality and use the fact that $u y=y-y u$ to obtain $2(x \cdot y) u+y x$ $=2(y u) \cdot x+2(y x) \cdot u$. Since $x \cdot y$ is in $N,(x \cdot y) u$ is in $N_{1}$ and since $(y u) \cdot x$ is in $N$, it follows that $y x-2(y x) \cdot u$ is in $N$. This implies that $\alpha=\beta=0$ and so $x y$ and $y x$ are in $N+A_{12}$.

Lemma 2. If $A$ is nilstable and if $B=A_{12}+N$ is an ideal of $A^{+}$, then $B$ is an ideal of $A$. Thus if $A$ is simple, $B=0$.

Since $B$ is an ideal of $A^{+}, x y+y x$ is in $B$ for every $x, y$ in $A_{12}$. By properties of the decomposition for $A^{+}$(see [1]), we also know that $x y+y x$ is in $A_{1}+A_{2}$. Therefore $x y+y x$ is in $N$. By Lemma $1, x y$ and $y x$ are in $B$. Using our hypothesis of nilstability and the facts that $N_{1}$ and $N_{2}$ are orthogonal subalgebras, it is now easy to verify that $B$ is an ideal of $A$.

Now we have Lemma 7 of reference [4] and all the results that follow it up to the fourth line from the end of the paper where we have that $C=A_{12} \cdot\left(N_{1}+N_{2}\right)+N_{1}+N_{2}$ is an ideal of $A^{+}$. Call the component of $A_{12}\left(N_{1}+N_{2}\right)$ in $A_{12}$ by $A_{12}^{*}$ and let ${ }^{*} A_{12}$ denote the $A_{12}$ component of $\left(N_{1}+N_{2}\right) A_{12}$. It will be shown that $D=* A_{12}+A_{12}^{*}+N_{1}$ $+N_{2}$ generates an ideal of $A$ which is properly contained in $A$. If $A$ is simple, this ideal will have to be the zero ideal, and thus $N_{1}=N_{2}=0$. Then $A=u F+A_{12}+v F$ and it is easy to verify that $A^{+}$is Jordan.

Lemma 3. Let $x$ be any element of ${ }^{*} A_{12}+A_{12}^{*}$ and $y$ any element of $A_{12}$. Then $x y, y x,\left(x R_{u}^{n}\right) y, y\left(x R_{u}^{n}\right),\left(y R_{u}^{n}\right) x$, and $x\left(y R_{u}^{n}\right)$ are in $N+A_{12}$ for every positive integer $n$, where $R_{u}$ denotes right multiplication by $u$.

Since $C$ is an ideal of $A^{+}, y(z g+g z)_{12}+(z g+g z)_{12} y$ is in $C$ for $y, z$ in $A_{12}, g$ in $N$. We emphasize once more that $(z g+g z)_{12}$ is the component of $z g+g z$ in $A_{12}$. Furthermore $y(z g+g z)_{12}+(z g+g z)_{12} y$ is in $A_{1}+A_{2}$, so it must be in $N$ for every $y$. By Lemma $1, y(z g+g z)_{12}$ and $(z g+g z)_{12} y$ are in $A_{12}+N$. From (1), $y(z g)+g(z y)=(y z) g+(g z) y$. This implies that $y(z g)_{12}-(g z)_{12} y$ is in $A_{12}+N$. Combining these results we have that $y(g z)_{12}+(g z)_{12} y$ and $y(z g)_{12}+(z g)_{12} y$ are in $A_{12}+N$ and since they are also in $A_{1}+A_{2}$, they are in $N$. Using Leinma 1 
again, $y(g z)_{12},(g z)_{12} y, y(z g)_{12}$, and $(z g)_{12} y$ are in $A_{12}+N$. Since any $x$ in ${ }^{*} A_{12}+A_{12}^{*}$ is a linear combination of elements of the form $(g z)_{12}$ and $(z g)_{12}, x y$ and $y x$ are in $A_{12}+N$ for any $y$ in $A_{12}$. The elements $y R_{u}^{n}$ are in $A_{12}$, so we also have $\left(y R_{u}^{n}\right) x$ and $x\left(y R_{u}^{n}\right)$ in $A_{12}+N$. The remainder of the lemma is proved by induction on $n$. Add $(x y) u$ to both sides of $(y x) u+(u x) y=y(x u)+u(x y)$ and use $u x=x-x u$ to get $2(x \cdot y) u+x y=2(x u) \cdot y+2 u \cdot(x y)$. It follows that $(x u) \cdot y$ is in $A_{12}+N$. This fact is for every $y$ so Lemma 1 implies $(x u) y$ and $y(x u)$ are in $A_{12}+N$. Replace $x$ by $x R_{u}^{n}$ in the above and use the induction hypothesis to obtain the facts that $\left(x R_{u}^{n+1}\right) y$ and $y\left(x R_{u}^{n+1}\right)$ are in $A_{12}+N$. This completes the proof of Lemma 3.

Lemma 4. If for some $x$ in $A_{12}, x y$ and $y x$ are in $A_{12}+N$ for every $y$ in $A_{12}$, then $(x y)_{12} z$ and $z(x y)_{12}$ are in $A_{12}+N$ for every $z$ in $A_{12}$.

Relation (1) gives $(x y) z+(z y) x=x(y z)+z(y x)$ and our hypotheses imply that $(x y)_{12} z-z(y x)_{12}$ is in $A_{12}+N$. But $x y+y x$ is in $A_{1}+A_{2}$ and thus $(y x)_{12}=-(x y)_{12}$. Therefore, $(x y)_{12} z+z(x y)_{12}$ is in $A_{12}+N$ and Lemma 1 gives the desired result.

Lemma 5. If for some $x$ in $A_{12}, x y$ and $y x$ are in $A_{12}+N$ for every $y$ in $A_{12}$, then $\left[(x y)_{12} R_{u}^{n}\right] z$ and $z\left[(x y)_{12} R_{u}^{n}\right]$ are in $A_{12}+N$ for every $z$ in $A_{12}$.

The proof is patterned after the last part of Lemma 3 and we omit the details.

Let $M=A_{12}^{*}+{ }^{*} A_{12}$ and let

$$
\begin{aligned}
B= & N+M+\sum_{k} M R_{u}^{k} \\
& +\left\{\sum_{k}\left(M R_{u}^{k}\right) A_{12}+\sum_{k, m}\left[\left(M R_{u}^{k}\right) A_{12}\right]_{12} R_{u}^{m}+\sum_{k}\left[\left(M R_{u}^{k}\right) A_{12}\right]_{12} A_{12}\right. \\
& +\sum_{k, m}\left(\left[\left(M R_{u}^{k}\right) A_{12}\right]_{12} R_{u}^{m}\right) A_{12}+\sum_{k, m}\left(\left[\left(M R_{u}^{k}\right) A_{12}\right]_{12} A_{12}\right)_{12} R_{u}^{m} \\
& \left.+\sum_{k}\left(\left[\left(M R_{u}^{k}\right) A_{12}\right]_{12} A_{12}\right)_{12} A_{12}+\cdots\right\}_{12}
\end{aligned}
$$

for $k, m$ any positive integers.

The dots indicate that we take all possible elements in $A_{12}$ which can be obtained by starting with an element in $M$ and multiplying this on the right in every possible way by powers of $R_{u}$ and by elements of $A_{12}$. There are actually only a finite number of types of products because $A$ is finite dimensional. Since $(a b)_{12}=-(b a)_{12}$ for any $a, b$ in $A_{12}$ it is unnecessary to consider left multiplication by ele- 
ments of $A_{12}$. Left multiplication by $u$ need not be considered either since $u a=a-a u$ for any $a$ in $A_{12}$. The preceding lemmas imply that $B$ is an ideal of $A$. Since $B \subseteq N+A_{12}, B$ does not contain $u$, and if $A$ is simple, $B=0$. It follows that $N_{1}=N_{2}=0$ and $A=u F+A_{12}+v F$. To prove that $A^{+}$is a Jordan algebra use the fact that $x \cdot y=\alpha(u+v)$ for every $x, y$ in $A_{12}, \alpha$ in $F$. (See Lemma 10 of [2].)

The result of Theorem 3 says that an algebra $A$ satisfying its hypotheses is a noncommutative Jordan algebra. A result of Oehmke (Theorem 4.1 of [5]) gives

THEOREM 4. Let $A$ be a nilstable simple flexible power-associative algebra of degree 2 over an algebraically closed field of characteristic $\neq 2,3$. Then $A^{+}$is a simple Jordan algebra.

Combining Theorems 2 and 4 with Theorems 2.7 and 4.1 of [5] we have the following result.

TheOREm $5 .^{2}$ Let $A$ be a simple flexible power-associative algebra of degree $t \neq 1$ over an arbitrary field of characteristic 0 . Then $A^{+}$is a simple Jordan algebra.

3. Description of the simple algebras. Over an algebraically closed field the simple Jordan algebra of degree $2, A^{+}$, can be described as follows. It has a basis $u, v, a_{1}, \cdots, a_{n}$ for some integer $n$ where $u$, $v$ are orthogonal idempotents such that $u+v=1$ is the unity element of $A^{+}, a_{i} \cdot u=a_{i} \cdot v=a_{i}, a_{i}^{2}=1$ for each $i$, and $a_{i} \cdot a_{j}=0$ if $i \neq j$.

To describe $A$ completely it is necessary to describe the products $u a_{i}$ and $a_{i} a_{j}$. These products determine $a_{i} u=a_{i}-u a_{i}, v a_{i}=a_{i}-u a_{i}$, $a_{i} v=a_{i}-v a_{i}$, and $a_{j} a_{i}=-a_{i} a_{j}$. Since $u a_{i}$ is in $A_{12}, u a_{i}=\sum_{j} \alpha(i, j) a_{j}$. Write $a_{i} a_{j}=\gamma u+\delta v+\sum_{k} \epsilon(i, j, k) a_{k}$. First we note that from the flexible law $\left(a_{i} u\right) a_{i}=a_{i}\left(u a_{i}\right)$ it follows that $a_{i}^{2}=\left(u a_{i}\right) a_{i}+a_{i}\left(u a_{i}\right)$ and therefore $1=2 \alpha(i, i)$. From (1) we have $\left(a_{i} u\right) a_{k}+\left(a_{k} u\right) a_{i}=a_{i}\left(u a_{k}\right)$ $+a_{k}\left(u a_{i}\right)$. Using $a_{i} u=a_{i}-u a_{i}, a_{i} a_{k}-\left(u a_{i}\right) a_{k}+a_{k} a_{i}-\left(u a_{k}\right) a_{i}=a_{i}\left(u a_{k}\right)$ $+a_{k}\left(u a_{i}\right)$. If $i \neq k, a_{i} a_{k}+a_{k} a_{i}=0$, and hence $2 \alpha(i, k)+2 \alpha(k, i)=0$. Using (1) again, $\left(a_{i} a_{j}\right) u+\left(u a_{j}\right) a_{i}=a_{i}\left(a_{j} u\right)+u\left(a_{j} a_{i}\right)$. Since $a_{j} a_{i}$ $=-a_{i} a_{j}$ and $a_{j} u=a_{j}-u a_{j},\left(a_{i} a_{j}\right) u+\left(u a_{j}\right) a_{i}=a_{i} a_{j}-a_{i}\left(u a_{j}\right)-u\left(a_{i} a_{j}\right)$. Thus $\gamma u+\left(u a_{j}\right) a_{i}+a_{i}\left(u a_{j}\right)=\delta v$ or $\gamma u+2 \alpha(j, i) 1=\delta v$. Consequently, $2 \alpha(j, i)=\delta=-\gamma$.

The substitution $x=a_{i}, y=a_{j}, z=a_{t}$ in (1) gives $\left(a_{i} a_{j}\right) a_{t}+\left(a_{t} a_{j}\right) a_{i}$ $=a_{i}\left(a_{j} a_{t}\right)+a_{t}\left(a_{j} a_{i}\right)=-a_{i}\left(a_{t} a_{j}\right)-a_{t}\left(a_{i} a_{j}\right)$ or $\left(a_{i} a_{j}\right) \cdot a_{t}+\left(a_{t} a_{j}\right) \cdot a_{i}=0$. Therefore $\epsilon(i, j, t)+\epsilon(t, j, i)=0$. Since $a_{j} a_{i}=-a_{i} a_{j}, \epsilon(i, j, t)=-\epsilon(j, i, t)$. Combining these statements we see that if $a, b, c$ is any permutation

${ }^{2}$ This is the only place in the paper where we do not assume that the degree of $A$ is two. From now on we shall always suppose degree $A=2$. 
of $i, j, t, \epsilon(a, b, c)=\epsilon(i, j, t)$ if the permutation is even and is the negative of $\epsilon(i, j, t)$ for an odd permutation. The above results are summarized in the following theorem.

THEOREM 6. Let $A$ be an algebra over an algebraically closed field of characteristic $\neq 2$ such that $A^{+}$is the simple Jordan algebra of degree 2. Then multiplication in $A$ is defined by $u a_{i}=\sum_{j} \alpha(i, j) a_{j}$ where $\alpha(i, i)$ $=1 / 2$ and $\alpha(j, i)=-\alpha(i, j)$ if $i \neq j$, and, for $i \neq j, a_{i} a_{j}=2 \alpha(i, j)(u-v)$ $+\sum_{k} \epsilon(i, j, k) a_{k}$ where $\epsilon(i, j, k)$ is a linear skew-symmetric function on $i, j, k$.

It is easy to see that the properties discussed above are sufficient to give simple, flexible, power-associative stable (since a Jordan algebra $A^{+}$is stable) algebras of degree 2 . That is, let $F$ be any field of characteristic not 2 , let $u, v, a_{1}, \cdots, a_{n}$ be a basis for a vector space $A$ over $F$ and define multiplication in $A$ by $u^{2}=u, v^{2}=v, u v=v u=0$, $u a_{i}=\sum_{j} \alpha(i, j) a_{j}$ with $\alpha(i, i)=1 / 2, \alpha(j, i)=-\alpha(i, j)$ are all in $F, a_{i}^{2}$ $=u+v$, and, for $i \neq j, a_{i} a_{j}=\sum_{k} \epsilon(i, j, k) a_{k}$ where $\epsilon(i, j, k)$ is in $F$ and is a linear skew-symmetric function on $i, j, k$. Then it is a straightforward matter to show that $A^{+}$is a simple Jordan algebra of degree 2 and that $A$ is flexible. The fact that $A$ is simple follows from the fact that $A^{+}$is simple.

\section{REFERENCES}

1. A. A. Albert, Power-associative rings, Trans. Amer. Math. Soc. 64 (1948), 552593.

2. - A theory of power-associative commutative algebras, Trans. Amer. Math. Soc. 69 (1950), 503-527.

3. L. A. Kokoris, New results on power-associative algebras, Trans. Amer. Math. Soc. 77 (1954), 363-373.

4. - Simple power-associative algebras of degree two, Ann. of Math. 64 (1956), 544-550.

5. R. H. Oehmke, On flexible algebras, Ann. of Math. 68 (1958), 221-230.

6. - On flexible power-associative algebras of degree two, Proc. Amer. Math. Soc. 12 (1961), 151-158.

ILLINOIS INSTITUTE OF TECHNOLOGY 